\title{
Asteroseismology of B and A stars combining Indian and South African resources
}

\author{
Chris Engelbrecht*
}

\begin{abstract}
Department of Physics, University of Johannesburg, PO Box 524, Auckland Park, 2006, South Africa
\end{abstract}

\begin{abstract}
Various strong and productive collaborations in diverse aspects of asteroseismology have been established between Indian and South African astronomers over the past 18 years. An important factor in this success has been the fusion of diverse capabilities in both instrumentation and research expertise. Past successes are very briefly summarised. A limited summary of new telescopes and instruments available in India and South Africa, respectively, is provided and future prospects are briefly summarised.
\end{abstract}

\section{Introduction}

The respective geographical locations of India and South Africa make a strong case for collaboration on observational campaigns targeted at groups or classes of objects that are scattered across the Galaxy. India has observing facilities that cover a large range of northern latitudes (the Indian Astronomical Observatory lies at 32 degrees North and the Vainu Bappu Observatory at 12 degrees North), while the primary observing sites in South Africa occur at southern latitudes of 32 degrees (Sutherland) and 29 degrees (Boyden). This allows coverage of a very large portion of the night sky through a combination of Indian and South African telescopes. Furthermore, the differences in longitude allow very long uninterrupted observations of objects closer to the celestial equator, which can be tracked from both Indian and South African sites.

A couple of joint Indo-South African projects have originated form these geographical considerations. Past successes are discussed in Sect. 2, while future prospects are discussed in Sect. 3 and final conclusions are presented in Sect. 4.

\section{Past successes}

Two past projects are discussed in this section: The Nainital-Cape survey of chemically peculiar stars and various campaigns related to the target field of the Kepler space telescope, including follow-up spectroscopy of apparently anomalous pulsating B and A stars detected with Kepler.

*chrise@uj.ac.za 


\subsection{The Nainital-Cape survey}

The goal of this joint project was to make a systematic and comprehensive search for oscillations in chemically peculiar stars, mostly Ap and Am stars. Observatories in the North (at ARIES, Nainital) and in the South (at SAAO, Sutherland) were conducted over the decade spanning 1999-2009, to obtain comprehensive sky coverage. Some of the milestone papers describing this project are Martinez et al. (2001) and Joshi et al. (2006, 2009, 2016). More than a dozen Indian astronomers and five South African astronomers collaborated on this project, as well as additional researchers from the UK and China. Indian astronomers include S. Joshi, Y. Joshi, R. Sagar, D.L. Mary, S. Seetha, V. Girish, B.N. Ashoka, U.S. Chaubey, S.K. Gupta, K. Kasturirangan, N.K. Chakradhari, S.K. Tiwari, C. Billaud and Y.B. Kumar. South African astronomers include P. Martinez, L.A. Balona, R.T. Medupe, H.P. van Heerden and R.B. Kuhn. Don Kurtz (UK) and A Zhou (China) also collaborated on this project. As summarised in Joshi et al. (2016), a total of 337 chemically peculiar stars were observed. A key finding of this campaign was that oscillations with sub-millimag amplitudes could be reliably detected at these sites, even with the small telescopes used (almost all of the surveyed targets had V magnitudes between 7 and 9, although a few targets were brighter than $\mathrm{V}=7$ and a few were fainter than $V=9$ ) . Besides detecting a few new pulsators among the chemically peculiar stars, another finding of interest was that the majority of chemically peculiar stars included in the survey did not display detectable oscillations, even at the very low detection thresholds attained. This finding will assist progress towards a full understanding of the dynamics of oscillations in chemically peculiar stars.

\subsection{Open cluster studies and spectroscopic follow-up of Kepler targets}

A second suite of Indo-South African joint projects originated in 2008, with the main aim of making follow-up observations of potentially interesting stars in the Kepler field of view. The first project explored the extent and nature of stellar variability in the open cluster NGC6866, which is in the Kepler field, through multi-colour photometry obtained at ARIES, Nainital (Joshi et al. 2012, Balona et al. 2013). An intensive follow-up study (Joshi et al. 2014) was conducted on NGC559 (not in the Kepler field of view). In all, five Indian astronomers (Y.C. Joshi, S. Joshi, B. Kumar, S. Mondal and R. Sagar) and one South African astronomer (L.A. Balona) collaborated on these projects. A third project in this collaboration originated in 2013. The Kepler space telescope allowed unprecedented precision in the detection of low-amplitude oscillations in stars across the Hertzsprung-Russel (HR) diagram. A small number of detected pulsators appeared in regions of the HR diagram where pulsations should be totally damped, according to current models. In order to explore the physical parameters of these stars more accurately (and determine whether their apparent anomalous pulsation behaviour was actually due to misclassification on the HR diagram), this project was designed to obtain spectra of the suspected anomalous stars. As the Kepler field of view lies deep into the northern hemisphere, these stars would be inaccessible to South African telescopes but well placed for observation from India. Eventually, spectra were successfully obtained with the Himalaya Faint Object Spectrograph and Camera (HFOSC) mounted on the 2.0-m Himalayan Chandra Telescope (HCT) operated by the Indian Institute of Astrophysics (IIA), Bangalore. Additional spectra were also obtained from the Special Astrophysical Observatory (SAO) of the Russian Academy of Sciences with the Main Stellar Spectrograph (MSS). The results of this project are described in Balona et al. (2016). Six Indian astronomers (Y.C. Joshi, S. Joshi, B.K. Sharma, G. Pandey, N.K. Chakradhari and B.P. Hema) and two South African astronomers (L.A. Balona and C.A. Engelbrecht) collaborated on this project, as well as Evgeny Semenko from Russia, David Mkrtichian from Thailand and Jim Nemec from the USA. An important finding from the spectra obtained with this project is that all 6 apparently anomalous stars showing $\gamma$ Doradus-type pulsations are indeed hotter than the blue edge of the instability strip of the $\gamma$ Doradus stars (see Dupret et al. (2004) and Xiong et al. (2016) for calculated instability 
strips for the $\gamma$ Doradus stars). This project therefore confirmed the status of these stars as "maverick" pulsators, possibly pointing to new physics that we need to learn about intermediate-mass convective core stars. Spectra were also obtained for two putative anomalously cool (from the perspective of Beta Cephei stars!) high-frequency p-mode pulsators detected by Kepler in its original and follow-up (K2) mode respectively. The spectra were obtained with HFOSC (see above) and confirm that at least one of these stars is very likely also anomalous and could be classified as a Maia star candidate.

In summary, the full suite of Indo-South African projects conducted described above have made important contributions to our knowledge of stellar pulsations in the upper main sequence (late $\mathrm{O}$ to early $\mathrm{F}$ stars). The total number of astronomers involved runs into the dozens and the particular strengths of the respective instruments in India and South Africa have been well utilised to serve the goals of these projects. The respective research strengths of the astronomers involved have also dovetailed admirably to produce the obtained results. Furthermore, the past 18 years of collaboration have allowed the collaborators to develop various teams who work well together and understand each other well. Given this successful venture in Indo-South African collaboration on asteroseismology, it is natural to explore how this could be further developed into the future. The next section briefly summarises a few prospects.

\section{Future prospects}

Exciting new telescopes and instruments developed both in India and in South Africa open the door to further collaborations on the asteroseismology of (mainly) B and A stars. A brief (and incomplete) summary of new telescopes and instruments follows.

In India, the new 3.6-m telescope at Devasthal will be available for both low-resolution and highresolution spectroscopy. Past projects described in the previous section have indicated how useful even low-resolution spectra can be. The Devasthal 3.6-m telescope will therefore allow spectroscopic follow-up on even relatively faint stars. This could play a major role in confirming temperatures and surface gravities of pulsators detected with ongoing ground-based surveys and new space telescopes respectively. High-resolution spectra could allow metal content to be determined - a question of particular importance in understanding stars that depend on the iron-group opacity bump for excitation and driving of pulsations. High-resolution spectra can also be used to analyse high-degree nonradial oscillations through the study of line profile variations. Also at Devasthal, the 1.3-m telescope would be ideal for multi-colour photometric studies that could allow pulsation modes (degree and order) to be determined. The interaction between pulsation and rotation of massive stars could be studied by employing the photometric capabilities of the 1.3-m telescope and the spectroscopic capabilities of the 3.6-m telescope.

Exciting new developments in South African instrumentation dovetail with those in India. The 1.9-m telescope at Sutherland has been equipped with a new grating spectrograph, which has a resolving power ranging from 700-6500, depending on the grating used. Studies of the same target with the old and new spectrographs indicate a 4-fold increase in throughput, i.e. a fourfold decrease in the time required to obtain a spectrum with a given signal-to-noise ratio (SNR). The telescope is now capable of obtaining spectra with a signal-to-noise ratio of 100 per pixel for stars with $\mathrm{V}=$ 11 , in less than 20 minutes. The same telescope can also be used for high-speed photometry with a new state-of-the-art photometer. A brand new, remotely-operated 1.0-m telescope has also just become operational at Sutherland (open time on this telescope is being offered from 1 September 2017). The telescope can carry either a wide-field camera, a high-speed photometer (the same design as on the 1.9-m telescope) or a spectrograph. These telescopes therefore offer capabilities similar to those described above for the Indian facilities at Devasthal.

The similarity in performance of the Indian and South African instruments described above makes 
future Indo-South African collaborations on all-sky survey projects (focused on particular questions in asteroseismology) very viable. Further South African facilities available for asteroseismological research include: i) the 11-m aperture SALT telescope, with low/medium/high-resolution spectroscopic capability and an imaging camera; ii) South African time on the three 1.0-m Las Cumbres Observatory (LCO) telescopes at Sutherland; iii) the 1.4-m Infrared Survey telescope at Sutherland. In addition to these facilities at the Sutherland site, a $1.5-\mathrm{m}$ telescope with photometric capability is available at Boyden Observatory.

\section{Conclusion}

Robust collaborations in asteroseismology have been established between Indian and South African astronomers over the past 18 years. Valuable scientific results have emerged from these collaborations, as reported in many papers, including the references appearing at the end of this report. The fusion of diverse strengths of both the instruments and the researchers involved in these collaborations will be sustained in future collaborations that are already underway, allowing both nations to continue to participate in the golden age of asteroseismology that has developed in recent years. These long-term and successful Indo-South African collaborations serve as a good example of what the Belgo-Indian Network on Astronomy and Astrophysics (BINA) could become.

\section{Acknowledgements}

Support from the Department of Science and Technology (South Africa), DST (India) and the University of Johannesburg is gratefully acknowledged.

\section{References}

Balona L. A., Joshi S., Joshi Y. C., Sagar R. 2013, MNRAS, 429, 1466

Balona L. A., Engelbrecht C. A., Joshi Y. C. et al. 2016, MNRAS, 460, 1318

Dupret M., Grigahcéne A., Garrido R., Gabriel M., Scuflaire R. 2004, A\&A, 414, L17

Joshi S., Mary D. L., Martinez P. et al. 2006, A\&A, 455, 303

Joshi S., Mary D. L., Chakradhari N. K., Tiwari S. K., Billaud C. 2009, A\&A, 507, 1763

Joshi S., Martinez P., Chowdhury S. et al. 2016, A\&A, 590, A116

Joshi Y. C., Joshi S., Kumar B., Mondal S., Balona L. A. 2012, MNRAS, 419, 2379

Joshi Y. C., Balona L. A., Joshi S., Kumar B. 2014, MNRAS, 437, 804

Martinez P., Kurtz D. W., Ashoka B. N. et al. 2001, A\&A, 371, 1048

Xiong D. R., Deng L., Zhang C., Wang K. 2016, MNRAS, 457, 3163 